guided by percentages alone they may often be led astray. The real value of a determination of the quantity of organic impurity in a water is, that by it a very shrewd notion can be obtained as to what has had access to that water."

However startling these statements may be to those who judge of the wholesomeness of a water by the amount of organic matter it may contain, we believe it to be none the less an accurate description of facts. It is within our knowledge that some of our most wholesome supplies sometimes contain an excess of organic matter, and that the waters which give rise to typhoid fever and other hardly less serious disorders are frequently just those which contain the least, the difference of course being that in the one case the organic matter is innocuous, in the other deadly.

Since, then, chemical analysis fails entirely to distinguish between these two kinds of matter, it may be thought to be a work of supererogation to have recourse to it at all. Not so, however, for what analysis fails to do directly it can to a large extent do indirectly. Organic matter in solution in water is more or less prone to oxidation, the highly putrescible matter of sewage being most so, and that derived from vegetation very much less so. Hence it follows that one would expect to find the oxidised nitrogen compounds in greater excess in the one case than in the other, and as a matter of fact that is just what we do find. Almost invariably, in all waters of acknowledged wholesomeness, the quantity of nitrates never exceeds a certain small amount, whereas in waters, such as polluted well and spring waters, that have given rise to illness, the oxidised nitrogen compounds, with other accompaniments of sewage, are to be found in excess. By means then of these oxidised nitrogen compounds we get collateral evidence throwing light on the nature and probable source of the contamination of which a mere percentage estimation of organic matter would fail to give the slightest indication.

The mistake has been hitherto that the discussion has been narrowed by looking at the question almost entirely from a chemist's point of view. It is, however, to the biologist that we must look chiefly for the future elucidation of the subject, and he has a field of the widest range, embracing much untrodden ground, for his investigations.

Putting on one side the specific poisons which through the medium of water are able each to generate, after its kind, diseases such as typhoid fever, it is highly probable, judging from what has already been proved to take place in analogcus cases, that dangerous organic matter is not poisonous as such, but acts by affording the pabulum for organisms which are able to set up putrefactive changes in the blood of the person drinking polluted water. Even the conversion of organic matter into nitrates is not a mere chemical process of oxidation, since we now know that the oxidation only takes place by the help of a distinct ferment.

In the inquiry as to how far organic matter is destroyed in rivers, it is clearly insufficient to rely, upon laboratory experiments in which diluted sewage is exposed only to the oxidising influence of air. This is entirely to ignore the agency of vegetation and of the vast army of organisms, identical with or allied to bacteria, which, being endowed with various functions of reorganisation, convert the carbon and nitrogen of organic matter into simpler inorganic compounds, these in turn to become the food of the more highly organised aquatic vegetation.

Whilst therefore duly recognising the practical help that chemistry can afford in the more limited scope that properly belongs to it, wc trust, in the interest of sanitary science, that the enunciation of the views of so distinguished a biologist as Prof. Huxley may have their due weight with those to whom these questions are ordinarily referred, and will tend to promote a better understanding

and more solid ground for agreement than has up to the present seemed possible.

Charles Ekin

\section{THREE YEARS' EXPERIMENTING IN MENSURATIONAL SPECTROSCOPY $Y^{1}$}

\section{By a NEW HaNd THEREAT}

\section{II.}

The Whole Solar Spectrum.--Could an observer, who had once made close acquaintance with the glories of symmetry resident in great $\mathrm{A}$ of the solar spectrum, when seen in the brightness of a southern noon-day, under a dispersion of $33^{\circ}$ and magnifying power of Io, ever remain content therewith?

Never! if a particle of soul belonged to him! for he would be imperiously constrained from that moment to feel that he must see the whole solar spectrum as it is given forth effulgently to the denizens of the south by a nearly zenith sun, before he died; or to what purpose would he have lived in a sun-illumined world?

Out, therefore, once more to Lisbon the experimenter and his Wife went in 1878 , with the important assistance again of the Pacific Steam Navigation Company of Liverpool; but now, armed with a rather different apparatus. There was indeed the same heliostat and there were all the prisms belonging to the aurora spectroscope ; but instead of each of them being looked through singly and successively, they were now used all together, set out in a curvilinear line several feet long on a large table, and looked through all at once; with telescope and collimator each 32 inches in focal length; with magnifying power of 20 , and a further prismatic method supplanting the usual employment of coloured glasses to prevent false glare in the field of view; and then what a new world was opened up to behold and admire !

Lines multiplied on lines and in a perfection of finish and refinement, sometimes of infinite thinness, sometimes remarkable power; and the classic fields of those more refrangible portions of the spectrum where the great spectroscopists of the age, Kirchhoff and Secchi, Lockyer and Janssen, Huggins and Young, have chiefly gained their laurels, as expounders of the constitution of the sun, were surveyed with respect and all admiration; but first, foremost, and beyond everything else, were the glories of the illimitable depths of solar colour; colour, the best leading index that has ever been invented yet, to simplify and facilitate the description of all spectrum place.

After having got completely rid of those usual attendant impurities in solar spectroscoping, viz., chemically coloured glasses used as shades, the large dispersions now employed enhanced rather than dulled the solar colours ; raised one's ideal of what colour in light can be, and gave, through near fifty gradations, a definite and ever-memorable colour-characterisation to as many portions of the whole spectrum.

In presence of such solar colours, it seemed to be a wilful ignoring of one's best and plainest faculties to speak of the spectrum colours as being only 3 , or 5 , or even 7 . They might indeed be rather spoken of as next to infinite in number ; or rather still, as being just so many as there are easily perceptible differences of spectral place; but for that law of locomotion of colour-bands within certain limits, already discovered by the experimenter in his absorption spectra, and found equally applicable to the solar spectrum. Confining therefore the number of colours to something which should give each of them a breadth, not likely to be overpassed by the locomotive effects + and - on their boundaries, the following table of fifteen spectral colours was prepared after much discussion and criticism of each individual member of it :-

\footnotetext{
I Cont:nued from p. $x 95$.
} 


\begin{tabular}{|c|c|c|c|c|c|}
\hline \multirow[t]{2}{*}{ General Distinctions. } & \multirow[t]{2}{*}{ Particular Colours. } & \multicolumn{2}{|c|}{$\begin{array}{l}\text { Wave-N umber Spectral Place, } \\
\text { normally. }\end{array}$} & \multirow{2}{*}{$\begin{array}{l}\text { Solar Lines within those Limits } \\
\text { of Place: }\end{array}$} & \multirow{2}{*}{$\begin{array}{l}\text { Chemical Flame, and Electric Spark, } \\
\text { Lines within the same Limits. }\end{array}$} \\
\hline & & Extends from & Reaches to & & \\
\hline \multirow{5}{*}{$\begin{array}{l}\text { Red end of } \\
\text { Spectrum. }\end{array}$} & Ultra-Red. & 25, oco & 30,000 & $\mathrm{x}$. & - \\
\hline & Crimson-Red. & 30,000 & $34, \infty 00$ & $\mathrm{Y}$ and $\mathrm{A}$. & Rubidium $x$ and Potassium $\alpha$ \\
\hline & RED. & 34,000 & $37, \infty 00$ & Little $a$ and great B. & Lithium $\alpha$, nearly. \\
\hline & Scarlet-Red. & 37,000 & 39,000 & Great C. & Scarlet Hydrogen Line. \\
\hline & Light-Red. & $39, \infty 00$ & $40,0 c 0$ & and $\alpha$ Band. & Light-Red Oxygen. \\
\hline \multirow{5}{*}{$\begin{array}{l}\text { Middle of Spec- } \\
\text { trum. }\end{array}$} & Orange. & 40,000 & 42,000 & $\alpha$ Band and Rain-Band. & $\begin{array}{c}\text { Carbo-Hydrogen's Orange Band, } \\
\text { and chief Oxygen Line. }\end{array}$ \\
\hline & Yellow. & $42, c 00$ & $44, \infty 00$ & D. & Sodium a. \\
\hline & CITRON. & 44,000 & 47,000 & Aurora's chief Line. & $\begin{array}{c}\text { Carbo-Hydrogen's Citron Band } \\
\text { of Lines. }\end{array}$ \\
\hline & Green. & $47, \infty 00$ & $51, \infty 00$ & $\mathrm{E}$ and little $b$. & $\begin{array}{c}\text { Thallium } \alpha \text { and C.-h.'s Green. } \\
\text { Giant Line. }\end{array}$ \\
\hline & Glaucous. & 51,000 & 55,000 & Little $c$ and F. & Glaucous Hydrogen. \\
\hline
\end{tabular}

\begin{tabular}{c|c|c|c|c|c}
\hline Blue. & 55,000 & 57,000 & Little $d$. & Cæsium $\alpha$ and $\beta$. \\
\hline $\begin{array}{c}\text { Violet end of } \\
\text { Spectrum. }\end{array}$ & Indigo. & 57,000 & 58,000 & Littie $e$ and little $f$. & Indigo Nitrogen Band. \\
\hline vIOLET. & 58,000 & 61,000 & Great G and little $g$. & Violet-Hydrogen Line. \\
\hline Lavender. & 61,000 & 65,000 & Little $h$ and great $\mathrm{H}^{1}$ and $\mathrm{H}^{2}$. & Lavender-Hydrogen Line. \\
\hline Gray. & 65,000 & 70,000 & - & -
\end{tabular}

The colour question settled, then came the measurement of the places of the lines seen therein and amongst. Each day the rather ragged train of some simple, some compound, prisms was set to minimum deviation for each of them in the part of the spectrum concerned, and from 100 to 200 or more lines per day were securely recorded day after day; until at last, after that long and laborious journey through all the colours and all the lines, not omitting to chronicle in appearance, as well as measured place, a single one amongst 2,000 , at last, like huge volcanoes throwing out pillars of black smoke streaked with vertical lines, the overpowering forms of $\mathrm{H}^{\mathrm{x}}$ and $\mathrm{H}^{2}$ hove in sight, and formed a fitting balance as well as contrast in the violet to great $A$ and its rhythmical predecessors in the ref.

But long, long before soundings were touched in the appearance of these two smoky giants, certain questions had to be wrestled with touching the terms in which all spectral places should be measured and published.
Full of desire to contribute data for theorists, the experimenter had indulged in the prospect of recording all line-places in terms of wave-lengths; and had even made his versatile, Robinson Crusoe sort of solar spectroscope, read its scales in numbers increasing as the wave-lengths of light do, from the violet towards the red end of the spectrum ; and also caused it to present the violet end towards the left, and the red towards the right hand, as with most of his predecessor's maps employing wavelengths. Further still, as he found it expedient to compare the solar spectral lines the was observing each day with the best maps and photographs he could collect, he applied a wave-length scale to each of them, made them all turn their violet ends leftward, and then tried to trace each line visible in the telescope through all its previous renderings or omissions by previous observers.

But oh! the difficulty of carrying that principle out fully, with anything more than a very few leading lines. The difference of the differences of a diffraction or wave- 
length scale, between one part of the spectrum and another, as compared with an average refraction or prism representation, viz., some sixteen times, was found to defy all accuracy by any ordinary pen or pencil, and to mislead or confound the eye, as to the mere physiognomy of groupings of the lines. Then, worse still, nature herself, and spectrum-forming nature too, was being fought against, in having scales increasing their numbers for dispersion one way, when the prismatic deviations which produced these dispersions were going the other way. So at last it was determined that whatever the scale a pure theorist may eventually prefer to put a few spectrum places in at last, for his own purposes, the spectrum observer, in order to observe well, quickly, and safely throughout the whole spectrum must have :-

I. A scale according to nature, as to the direction of increase of its numbers.

2. Increasing therefore these numbers from red to violet, both because the prismatic deviations do the samc, and because, when the temperature of bodies is gradually raised, from that of the air in which we live up to such point that they begin to be luminous, the first light given off is red; and they only attain to violet light in the latest and most extreme degrees of heat eventually obtained.

3. Red therefore being the natural beginning of the spectrum, and all spectral numbers arranged as above, increasing towards the rest of the spectrum, the said red end requires to be placed on the left hand, so that every spectrum map may be told off as all writing and printing is made to read in all European countries, viz. from left to right, never from right to left.

4. Seeing that prisms will always be employed by some observers of the solar spectrum, and gratings by others, the scale to be used should be one whose general form, in equal parts, should divide the immense difference of physiognomy which exists between the spectra offered by these two instrumental methods; that is, not compressing the red end so much as the prism does, nor compressing the violet so much as the grating does; and this end is obtained most neatly, on an equally absolute foundation with wave-lengths, and in a handy set of whole figures by adopting the number of such waves to the inch, British.

The above points having been all fairly arrived at, after great sacrifices of both time and labour in the other direction, the Edinburgh experimenter proceeded without any further compunction to alter his spectroscore once more, and make it conform in all respects thereto, i.e., to show the red end of spectra towards the left, and to increase spectral readings from left to right; while he further applied new scales to his collection of spectrum maps in terms of wave-numbers. And then came the reward; for not only did the same eye and pencil succeed in applying a wave-number scale more accurately than a wave-length one to prism-observed spectra, and make the correspondences between prism and grating spectra more numerous, perfect, and easily apprehensible, but the wave-number scale was found more suited naturally to the absolute requirements of the solar spectrum in itself. Or thus, while the wave-length scale, as represented in Angström's grand normal solar, but diffraction spectrum stretches out the red end to such a degree that the lines there are so few and far between as to waste the very paper on which they are drawn, the wave-number method gently compresses them, or brings them twice as close together; while again, if at the violet end the lines are so numerous, and closed packed in Ångström's map that they have hardly standing room, and can scarcely be separated one from the other-the wavenumber method gives them twice as much space there, in a map measuring, on the whole, from red to violet, only the same length as Ängström's.

But there was a still higher reward to the experimenter, who, adopting the scale of wave-number, and finding he had more room for the violet end of the spectrum, began to pay more attention thereto; for he then found that, crowded as were the violet lines in Angström's diffraction map, they were not half crowded enough; or rather that there were really in that part of the solar spectrum three or four times as many more lines still; far more indeed than could have been inserted on the engraved plates of the Swedish philosopher, and many more than his diffraction grating was probably able to show. While therefore all strong lines throughout Ångström's map are believed to have been most admirably measured, and the far more numerous thin lines are also most truthfully rendered in the earlier and middle parts of the spectrum-the violet termination, what with the imperfect showings of his grating, and the contracted space of the wave-length scale map, has not been done justice to.

Yet this is a very material point in the physics of the sun; for according to the preponderance of violet, over red, light, so may be assumed the intensity of the temperature of that light's origin. Whereabouts then did the increased number of lines in the violet observed by the Edinburgh experimenter with his prisms, over Angström with his grating, place the photosphere of the sun as to temperature?

This point, described by the experimenter in the Transactions of the Royal Society, Edinburgh, vol. xxix., for 1879 , was approximated to by him in this manner :Having collected from various sources several thousands of spectrum place observations, he reduced them all to wave-number scale, and then arranged them according to the temperatures of their sources of origin, or, as $\mathrm{Mr}$. Norman Lockyer has since then termed it, their respective "heat-levels," and the following series was obtained :-

Source of origin of spectral light, when at freezing point as in telluric absorption spectra, has its

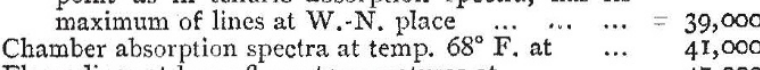
Flame lines at lamp-flame temperatures at $\quad \ldots \quad \ldots \quad 47,000$ Gas-vacuum tubes illuminated by $I$ inch induction spark

Chemical lines in $2{ }_{2}$ inch sparks $\cdots$ Chemical lines in 6 to 10 inch sparks intensified $\ldots . . \quad 51,000$ And Ångström's diffraction solar spectrum $\quad \ldots \quad \ldots \quad 55,000$

But the solar spectrum, as observed on this occasion in Portugal, showed its maximum of lines at $6 \mathrm{r}, 000$ of the same scale ; or indicated that the temperature of the solar photosphere may be as much above the highest temperature yet attained by man, even with assistance of electricity in its condensed form, as that is above the freezing temperature of the upper strata of the earth's atmosphere.

Lastly, Gaseous Spcctra.-Under this term are included both flames, especially blow-pipe flames, in the open air and electric illuminations inside so-called gas-vacuum tubes, such as those of Geissler and Plucker combined. But in all these cases the experimenter, finding that faintness of the light was the crying evil, changed the usual transverse method of looking at lines, or cones, of

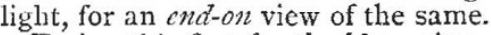

Trying this first for the blow-pipe, whose flame of coalgas urged by a stream of air could then, by a collimating objective applied to the anterior telescope, be safely looked into, though directed right towards the slit--the increased number of lines, their steadiness and definiteness in all the several hydro-carbon bands-and then the resolving of the mere haze in the field of view into closely ranked little lines or linelets, proved an inimitable reward, as well as a priceless source of the best kind of referencedata in all his subsequent inquiries; especially too because these advanced results were procured without increasing either the temperature, or size, or combustion material of the flame at all.

Next applying the same principle to the GeisslerPlucker tubes, by having their form modified by $M$. 
Saleron, so that they could be similarly looked at in the direction of the long line of the capillary-the effects were found almost startling in the brilliancy of the principal lines (chiefly indeed at the red end of the spectrum, for only weak sparks were employed) and in the immense number of additional lines in almost every tubespectrum examined. These results had been communicated to the Royal Scottish Society of Arts in I879, before it was ascertained that similar tubes for end-on use in photographing the violet lines had been made by the eminent Dr. van Monckhoven, at Gand, Belgium, three years earlier. But while fully acknowledging the Doctor's undoubted priority of invention, and inviting him to communicate his first published results at one of their meetings, the Society found the case already before them a perfectly independent invention; a part, too, of a more general system, and accompanied by a series of measures of some of the gas spectra, both in blowpipe flames and spark-illumined tubes, to a greater refinement than had ever been made before. They therefore graciously crowned the paper with a prize and printed it at full length in their Transactions for March, 1879 .

Now some of these increased refinements in knowledge of the spectra of the gases referred to matters long in dispute before the world; and especially to the contention of whether the so-called "carbon-lines" of some observers seen by them in candle-flames, could possibly be the lines of that most refractory element carbon, or were not rather the lines of some of the very easily volatilised compounds of carbon, unless all the usual chemistry of carbon be utterly at fault. Herein the powers of the aurora spectroscope with its bright images, its still brighter end-on methods of viewing gas-flames, and its easy powers of rotation from one source of light to another, proved of inestimable advantage; for not only could large dispersions, approaching those employed on the sun, be used with effect, but the minutest line in one spectrum could be so quickly compared with a similar line in any other, and decided on absolutely as to whether it was or was not in the same spectrum place.

Wherefore the Edinburgh experimenter proceeded in the following manner: after repeating Prof. Swan's ancient observations and finding with him that all the various hydrocarbons gave more or less completely the same spectrum as the blue base of a candle-flame does, he set up for permanent reference, end-on, a blowpipe flame of coal-gas with common air as the best example of that kind of spectrum, viz., the spectrum of a something which vapourises at merely lamp-flame temperature. That that thing could be pure carbon, the chemists one and all declare is impossible, because no furnace heat can vapourise that element; but the Royal Society, London, had printed a paper declaring that the unknown agent must be carbon, pure and elemental, because the author of that paper had seen the same spectrum, not only in all combinations of hydrogen with carbon, but in those of oxygen, and also nitrogen, with carbon. This statement too was further strengthened by a Report from the Greenwich Observatory in 1877 , to the effect that gas-vacuum tubes electrically illuminated, having been examined there spectroscopically, no sensible or material differences were found between carbo-hydrogens, carbooxygens, and carbo-nitrogens ; the one common spectrum seen there must also, it was argued, though very different from the blowpipe flame spectrum, be the spectrum of pure carbon.

But as soon as the Edinburgh experimenter tried his end-on vacuum tubes he found an immense difference between carbo-hydrogens on one side, and both carbooxygens and carbo-nitrogens on the other; for the former, though with some other features constant, invariably showed many most brilliant lines in the orange, the citron, the green, and the blue ; while the other tubes either had not any trace at all of those lines, or only so faint a mark- ing as to indicate they were there as impurities and not as the whole contents of the so-called "vacuum-tube."

What were these lines then, so peculiar to carbo-hydrogen tubes? A reference to the coal-gas blowpipe flame showed that they were its characteristic lines; the lines, too, of an easily dissociable compound gas therein, and not of an ultimate and most refractory element; for as soon as the electric sparks illuminating the tubes were somewhat increased in intensity, quantity, and heat, these blow pipe, or we may now safely call them carbo-hydrogen, lines faded out of view; while the two elements which had made them, viz., pure hydrogen, showed its lines, and pure carbon showed, not its ultimate, elemental lines (which nothing short of the most powerful sparks, large batteries, and enormous condensers far above the private means of the Edinburgh experimenter can bring forth), but its low-temperature, compound-linelet, or band, spectrum.

Next, on examining the tubes of carbo-oxygen and carbo-nitrogen certain differences between them were detected, due apparently to the compound gas in each case being partly dissociated, and partly left untouched, by the simple, small induction-sparks employed. When largely dissociated, then carbon bands and oxygen lines were grandly present in one case, and carbon bands and nitrogen bands in the other; with some indications also of the compound's presence in either case, though never to the magnificent degree of the carbo-hydrogen in tubes of that gas. This, however, was merely for the simple reason that carbo-hydrogen is by nature a more magnificent "lighter-up" in luminous spectra; just as it is indeed the basis of all the means yet adopted in the history of mankind to correct the darkness of night; and there seems little chance that science will ever find anything better for every kind of occasion wherein we now employ candles, gas-lights, and lamps.

On further examining the carbon bands in the end-on tubes by a dispersion power of $33^{\circ}$ from $\mathrm{A}$ to $\mathrm{H}$, a peculiar structure was discovered by the experimenter in their component lines; and when he found that to be as distinct in a cyanogen tube which contained no trace of either oxygen, or more unusual still, hydrogen impurity, he considered it a proof that that electric spark-raised carbon-band (to which the chemists will probably not object) was the low-temperature spectrum of that element, and not the spectrum, as argued by M. Thalen, of an oxide of carbon.

Many important points, therefore, seem to be indicated by these experiments, but with the general effect also of showing that spectroscopy loses much of its exceeding accuracy in power of discrimination, unless its observation be accompanied by some record of the particular "heat-level" at which the materials examined by it were rendered incandescent.

Hence a paper on these subjects was communicated to the Philosophical Magazine, London, in August, 1879; and further observations are now being carried on by the same experimenter on a new variety of his end-on tubes, prepared also by M. Salleron, and giving still brighter spectra than before, with the same electric illumination.

But all this is only while waiting for the aurora to appear, that phenomenon being the proper cynosure of this particular Edinburgh spectroscope. And now all men trust that the said aurora is soon to reappear, as the multifarious solar activities of a new sun-spot cycle have so evidently begun in the increased size and number of these spots ever since October, 1879; when they were critically considered, and openly announced in NATURE, to have at last shaken off the languor of their long minimum epoch, and to have begun in earnest their preparation for the new series now fairly under way.

(Since the above paper was written, the first of the new cycle of auroras to come, has been caught. See NATURE, vol. xxi. p. 492). 